\title{
Tecnura
}

\section{Modelación de la producción primaria fitoplanctónica en el humedal Jaboque, Bogotá D.C.}

\author{
Modeling of primary production of phytoplankton \\ in the wetland Jaboque, Bogotá D.C.
}

\author{
Julio Eduardo Beltrán Vargas ${ }^{1}$
}

Fecha de recepción: 4 de octubre de 2014

Fecha de aceptación: 18 de septiembre de 2015

Cómo citar: Beltrán Vargas, J. E. (2016). Modelación de la producción primaria fitoplantónica en el humedal Jaboque - Bogotá D.C. Revista Tecnura, 20(47), 85-95. doi: 10.14483/udistrital.jour.tecnura.2016.1.a07

\section{Resumen}

Se presenta un modelo de simulación dinámico para explicar el comportamiento general de la producción primaria fitoplántonica en el humedal de Jaboque - Bogotá D.C., Colombia, en tres secciones con características físicas y químicas diferenciales. El modelo tiene en cuenta las variables fisicoquímicas, área de la cubeta, profundidad, precipitación media anual, temperatura del agua, $\mathrm{pH}$ y concentración de clorofila_a. La modelación dinámica se basó en ecuaciones diferenciales y se utilizó el método Euler de integración, la modelación se desarrolló utilizando el programa computacional Stella $9.1^{\circledR}$. El modelo permite cuantificar la producción primaria fitoplántonica del humedal de Jaboque a partir de la concentración promedio mensual de clorofila_a, para cada sección. Los resultados de la modelación de la Ppf evidencian que las variaciones de la concentración de la Ppf en cada sección del humedal siguen un patrón inverso al comportamiento bimodal de la precipitación. Se encontró un alto grado de correspondencia entre los valores de clorofila_a de campo y la Ppf modelada de la siguiente manera $r^{2}=0.86$ para la primera sección y $r^{2}=0.86$ y $r^{2}=0.79$ para las secciones restantes. La determinación del error relativo fue de 0,57 , para la primera sección y 0,$35 ; 0,46$, indicando que los resultados no están sobrestimados. El modelo muestra en términos generales los aspectos funcionales del comportamiento de la Ppf y su relación con el proceso de eutroficación, y permite hacer recomendaciones para el manejo y restauración del humedal.

Palabras clave: humedal, Jaboque, modelación, dinámica, producción fitoplanctónica

\begin{abstract}
A dynamic simulation model is presented to explain the general behavior of the primary production of phytoplankton in the wetland Jaboque-Bogota, Colombia, in three sections with differential physical and chemical characteristics. The model takes into account the physicochemical variables, the basin area, depth, annual rainfall, water temperature, $\mathrm{pH}$ and concentration of chlorophyll_a. The dynamic modeling is based on differential equations and the Euler integration method is used, the modeling was
\end{abstract}

1 Biólogo Marino, magister en Planificación y Administración del Desarrollo Regional, máster en Ecoauditoría y Gestión Ambiental Empresarial, doctor en Ciencias - Biología. Docente de la Universidad Distrital Francisco José de caldas. Bogotá, Colombia. Contacto: jebeltran@ udistrital.edu.co 
developed using Stella $9.1 \circledR$ computer program. The model allows quantifying the primary production of phytoplankton in wetland Jaboque from chlorophyll _a monthly average concentration for each section. The results of the Ppf modeling show that Ppf concentration variations in each section of the wetland follow a reverse pattern to the bimodal behavior of precipitation. A high degree of correspondence between the values of chlorophyll_a Ppf field and modeled in the following manner $r^{2}=0.86$ for the first section and $r^{2}=0.86$ and $r^{2}=0.79$ for the remaining sections was found. Error determination was 0,57 relative to the first section and 0,$35 ; 0,46$, indicating that the results are not overstated. The model shows in general terms the functional aspects of behavior Ppf and its relation to the process of eutrophication, and it allows recommendations for the management and restoration of wetlands.

Keywords: dynamic, modeling, Jaboque, production of phytoplankton, wetland

\section{INTRODUCCIÓN}

En los lagos la producción primaria puede considerarse de dos modos: la que se da dentro del mismo lago (producción primaria autóctona) y la que llega al sistema luego de haber sido sintetizada en áreas diferentes (producción primaria alóctona). En los ecosistemas acuáticos, las microalgas y las cianobacterias constituyen el primer eslabón de la cadena trófica (Grimshaw et al., 1997). En su condición de productor primario, el fitoplancton permite la entrada de energía al sistema (Gómez, Larduet y Abrahantes, 2001). La producción primaria está determinada por la cantidad de luz, $\mathrm{CO}_{2}$ y la variación de formas nitrogenadas y fosfatadas. Según la cantidad de nutrientes disueltos, los ecosistemas acuáticos pueden ser calificados como oligotróficos, con pocos nutrientes $y$, por tanto, con baja producción primaria, o en el otro extremo, hipereutróficos, en donde la generación continua de biomasa, principalmente de fitoplancton, genera problemas en los flujos energéticos
(Contreras, Castañeda y Torres, 1997). En los humedales, donde el flujo de agua es mínimo, la mayoría de la materia orgánica en partículas permanece cerca de o en los sitios de producción, y los nutrientes son reciclados y retenidos por la microbiota allí presente a medida que la materia orgánica se descompone (Grimshaw et al.,1997). La cantidad de clorofila es proporcional a la cantidad de fitoplancton y esta es a su vez proporcional al estado trófico del cuerpo de agua. Como tal, la concentración de clorofila_a (Ryding y Rast, 1989) y la producción primaria fitoplántonica (Hakanson y Boulion, 2003) pueden ser usadas como indicadores apropiados para determinar el grado de eutroficación en lagos y en humedales (tabla 1).

Los valores de la producción primaria fitoplántonica son medidos en unidades de energía o masa de carbón orgánico $(\mathrm{C})$ y se utiliza frecuentemente para estimar la productividad biológica o el estado trófico de los cuerpos de agua (Hakanson y Boulion, 2002). La cantidad de clorofila_a en el agua es correspondiente con la cantidad de fitoplancton

Tabla 1. Estado trófico basado en la concentración de clorofila_a y la producción primaria

\begin{tabular}{lcc}
\hline \multicolumn{1}{c}{ Estado trófico } & $\mathbf{C h l} \mathbf{a} \mathbf{~ / ~} \mathbf{~ g} / \mathbf{m}^{\mathbf{3}}$ & Producción primaria fitoplancton $\mathbf{~} \mathbf{g C} \mathbf{~ m}^{\mathbf{3 *} \mathbf{a n ̃ o}}$ \\
\hline Oligotrófico & $<2,5$ & $<30$ \\
\hline Mesotrófico & $2-8$ & $25-60$ \\
\hline Eutrófico & $6-35$ & $40-200$ \\
\hline Hipertrófico & $30-400$ & $130-600$ \\
\hline
\end{tabular}

Fuente: Boulion y Hakanson (2003). 
(Hakanson y Peters, 1995; Wetzel, 2001; Gocke, Mancera, Vidal y Fonseca, 2003). La cantidad de fitoplancton es proporcional al estado trófico de los cuerpos de agua. Se puede utilizar la concentración de clorofila_a y su equivalente en $\mathrm{mgC} / \mathrm{m}^{3}$ para establecer la producción primaria fitoplantónica. Para estimar la producción primaria fitoplántonica del humedal Jaboque se elaboró un modelo de simulación dinámica basado en el principio de balance de masas, el cual permite relacionar la concentración de clorofila_a con la producción primaria fitoplántonica expresada en unidades de C mg/ $m^{3}$ (Hakanson y Boulion, 2003; Rivera, 2006).

\section{METODOLOGÍA}

\section{Área de estudio}

El humedal Jaboque se ubica en la localidad de Engativá, en el occidente de la ciudad de Bogotá, Colombia (figura 1); está contiguo a la cuenca del río Juan Amarillo, entre el Aeropuerto Internacional El Dorado y la Autopista Medellín. Abarca aproximadamente 57 ha y presenta una forma alargada en dirección sur-oriente-noroccidente.

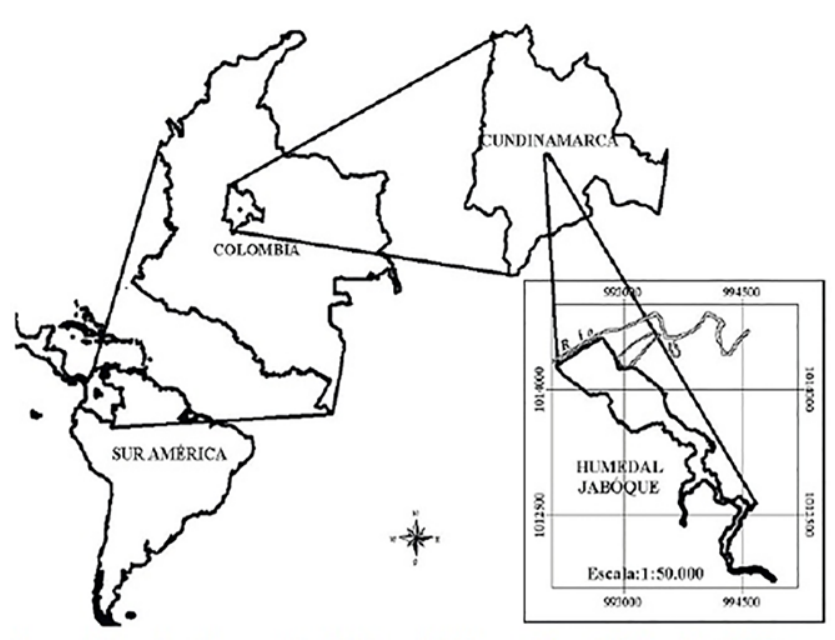

Figura 1. Localización geográfica del humedal Jaboque Fuente: Sierra y Monsalve ( 2005).
En las últimas cuatro décadas, el humedal Jaboque ha visto reducida su extensión por la variación del drenaje, la colmatación por cargas orgánicas de las aguas y vertimientos, ocupación por construcción y su fraccionamiento. Esta situación ha incidido para que el humedal esté perdiendo, por una parte, su función ecosistémica, y por la otra, sus atributos como: biodiversidad, estructura, procesos y funciones.

\section{Método de simulación}

La modelación se realizó con el software SteIla 9. $1^{\circ}$, utilizado en modelaciones hidrológicas y ecológicas (Jorgensen y Bendoricchio, 2001; Zhang y Mitsch, 2005; Jorgensen, 2008). Se trabajó el sistema de interfaz, para predecir el comportamiento de productividad primaria a partir de las concentraciones promedio de clorofila_a para cada sección; también para realizar los análisis de sensibilidad del modelo relacionando la precipitación con la productividad primaria fitoplantónica y así poder determinar el estado trófico del humedal (tabla 2). El modelo conceptual se transformó mediante algoritmos. En cada variable se desarrollaron las respectivas ecuaciones para establecer la relación entre las variables moderadoras, independientes y de estado del modelo. La simulación tuvo por objeto conocer y predecir el comportamiento de las fluctuaciones de la producción primaria fitoplantónica (Ppf) y estado trófico del humedal en sus tres secciones. Con el fin de verificar el carácter determinístico del modelo y realizar su validación, se llevaron a cabo regresiones simples lineares (Legendre y Legendre, 1998; Rivera, 2006), entre los valores de clorofila_a y la Ppf que permitieron comparar los valores estimados según análisis de laboratorio con los resultados modelados. Para evaluar la fortaleza del modelo, se determinó el error relativo (Bryhn y Hakanson, 2007; (Jorgensen y Bendoricchio, 2001; Zhang y Mitsch, 2005; Hakanson, 2004) y para evidenciar el carácter predictivo se analizó la sensibilidad del mismo modelo (Hakanson, 2004; Jorgensen, 2008) . 


\section{Variables del modelo}

Tabla 2. Variables utilizadas en el modelo

\begin{tabular}{|c|c|c|}
\hline Variables e índices & Valores/Unidades & $\begin{array}{l}\text { Fuente } \\
\end{array}$ \\
\hline Profundidad sección & $\mathrm{m}$ & $\begin{array}{l}\text { Instituto de Ciencias Naturales - Grupo de investigación Biodiver- } \\
\text { sidad y conservación - Universidad Nacional de Colombia }\end{array}$ \\
\hline Precipitación & m.m/año & $\begin{array}{l}\text { Instituto de Hidrología, Meteorología y Estudios Ambientales } \\
\text { (Ideam) }\end{array}$ \\
\hline Área sección & $\mathrm{m}^{2}$ & $\begin{array}{l}\text { Instituto de Ciencias Naturales - Grupo de investigación Biodiver- } \\
\text { sidad y conservación - Universidad Nacional de Colombia }\end{array}$ \\
\hline Volumen & $\mathrm{mm}$ & Datos de campo U. Nacional/Simulación \\
\hline Temperatura & Centígrados & $\begin{array}{l}\text { Instituto de Ciencias Naturales - Grupo de investigación Biodiver- } \\
\text { sidad y conservación - Universidad Nacional de Colombia }\end{array}$ \\
\hline Concentración de clorofila_a & $\mathrm{mg} / \mathrm{m}^{3}$ & $\begin{array}{l}\text { Instituto de Ciencias Naturales - Grupo de investigación biodiver- } \\
\text { sidad y conservación - Universidad Nacional de Colombia }\end{array}$ \\
\hline Tasa renovación fitoplancton & $\mathrm{Kg} \mathrm{wwC} / \mathrm{m}^{3} / \mathrm{t}^{-1}$ & Hakanson y Boulion (2003) \\
\hline Tasa de producción de fitoplancton & $\mathrm{mgC} / \mathrm{m}^{3} / \mathrm{t}^{-1}$ & Hakanson y Boulion (2003) \\
\hline Concentración de fitoplancton & $\mathrm{mgC} / \mathrm{m}_{*}^{3}$ año & Simulado \\
\hline Producción primaria inicial & $\mathrm{kg} w w \mathrm{C} / \mathrm{m}^{3}{ }_{*}$ año & Simulado \\
\hline $\begin{array}{l}\text { Producción primaria fitoplántonica } \\
\text { biomasa }\end{array}$ & $\mathrm{kg} w w \mathrm{C} / \mathrm{m}^{3}{ }_{*}$ año & Simulado \\
\hline
\end{tabular}

Fuente: elaboración propia.

\section{Supuestos del modelo}

El modelo asume los siguientes supuestos, con el propósito de delimitar su alcance y posterior generalización o aplicación:

- La carga (Ci) de de clorofila_a se calcula a partir del punto de entrada de cada sección.

- Los datos de Chl_a (Clorofila a) disponibles corresponden a un año de muestreo, que representa periodos hídricos invierno-verano (Álvarez, 2005).

- En la modelación del estado trófico del humedal se tuvo en cuenta la concentración de Chl_a.

- La modelación consideró los promedios mensuales de Chl_a encontrados en las zonas de flujo de entrada a cada sección.

- De acuerdo con la zonificación del humedal Jaboque, se asumen tres secciones con características morfométricas, físicas y biológicas propias.

- El modelo hace referencia a la producción primaria fitoplantónica.

\section{El modelo de simulación}

A partir de la relación entre la concentración de clorofila_a y la producción primaria fitoplantónica (calculada como $\mathrm{mgC} / \mathrm{m}^{3} / \mathrm{año}$ ), se calculó la producción primaria fitoplantónica y de acuerdo con Boulion y Hakanson (2003) en la ecuación (1) se trabajaron los siguientes algoritmos:

$$
P p f=\left(2,13 * C h l_{-} a^{0,25}+0.25\right)^{4}
$$

Donde,

$P p f=$ producción primaria fitoplantónica calculada como $\mathrm{mg} / \mathrm{m}^{3} / \mathrm{mes}$

Chl_a = concentración de clorofila a en $\mathrm{mg} / \mathrm{m}^{3}$.

Realizando la transformación logarítmica, según las ecuaciones (2), (3), (4) y (5) (Boulion y Hakanson, A ; 2003) 


$$
\begin{aligned}
& \log (P p f)=0,97 \cdot \log * \log \left(C h l_{-} a+2,49\right) \\
& P p f=30,6 * C h l^{0,927} \approx 30 * C h l_{-} a \\
& B m(t)=B m_{u}(t-d t)+(I P R-E l) * d t
\end{aligned}
$$

$B r=$ biomasa de producción primaria por unidad en $\mathrm{kg} \mathrm{w}$

$I P R=$ producción primaria inicial en $k g w w *$ semana

$E I=$ tasa de renovación de organismos consumidores $k g$ ww*semana (Boulion y Hakanson, 2003).

$$
P p f=\left(30,6 * C h l_{a}^{0,927}\right) * 0,45 * 30 * \text { Área } * Z * 0,01 *\left(\frac{R W T+0,1}{17}\right) * \frac{B M p}{B M f}
$$

$P p f=$ producción primaria inicial $(k g w w /$ semana).

$C h l=$ promedio mensual de concentración de clorofila_a $\left(\mathrm{mg} / \mathrm{m}^{3}\right)$.

Chl_a $=0,927^{*} 30,6=$ patrón de conversión de $C h /$ en producción primaria fitoplantónica en $\mathrm{Cmg} / \mathrm{m}^{3} / \mathrm{d}$.

0,45 = factor de conversión estándar para transformar Cg semana a $g d w / m e s$.

Área = área de la cubeta.

$Z$ = profundidad promedio.

$R W T=$ temperatura media de referencia.

$B M p / B M f=$ relación entre la biomasa de las entradas de plancton y la biomasa calculada de fitoplancton. El rango de esta relación es aproximado a 0,25 (Boulion y Hakanson, 2003).

\section{ANÁLISIS DE RESULTADOS}

\section{Comportamiento de la clorofila_a en el humedal de Jaboque}

Las variaciones de la concentración de la clorofila_a en cada sección del humedal siguen un patrón inverso al comportamiento bimodal de la precipitación. En los meses secos las concentraciones aumentan, mientras que en los meses lluviosos tienden a descender. Las dos primeras secciones muestran las mayores concentraciones de clorofila_a, y en la tercera sección las concentraciones de clorofila_a tienden a disminuir, resultado que se debe al hecho de que la primera y segunda sección reciben con mayor fuerza la descarga de nutrientes de la cuenca aferente (Fraile, Orive y Pozo, 1995; Boulion y Hakanson, 2003) (figura 2 y tabla

$3)$. Estos resultados sugieren que las condiciones climáticas y el hidroperiodo influyen en el comportamiento de la clorofila_a como lo sugieren Ryding y Rast (1989). En el mismo sentido, Jiménez (2006) encontró para el lago de Guatavita, (Cundinamarca, Colombia) variaciones de clorofila_a debidas a la radiación solar; sin embargo, estas no fueron ocasionadas por el hidroperiodo. Montoya y Aguirre (2010) en la ciénaga Escobillitas (Antioquia) hallaron una relación entre la variación los clorofila_a, la producción primaria y el pulso de inundación.

\section{Producción primaria fitoplantónica}

Los resultados de la modelación de la producción primaria del fitoplancton en el humedal Jaboque $\left(\mathrm{mgCm}^{-3}\right)$, para todas las secciones, se presentan en la figura 3 y en la tabla 3 . Se observa que la producción primaria aumenta en los periodos de menores precipitaciones (diciembre-febrero y julio-septiembre) y en los periodos de altas precipitaciones disminuye (abril, mayo, junio y octubre, noviembre). Es innegable una fuerte relación entre la actividad biológica y el ciclo hidrológico. 


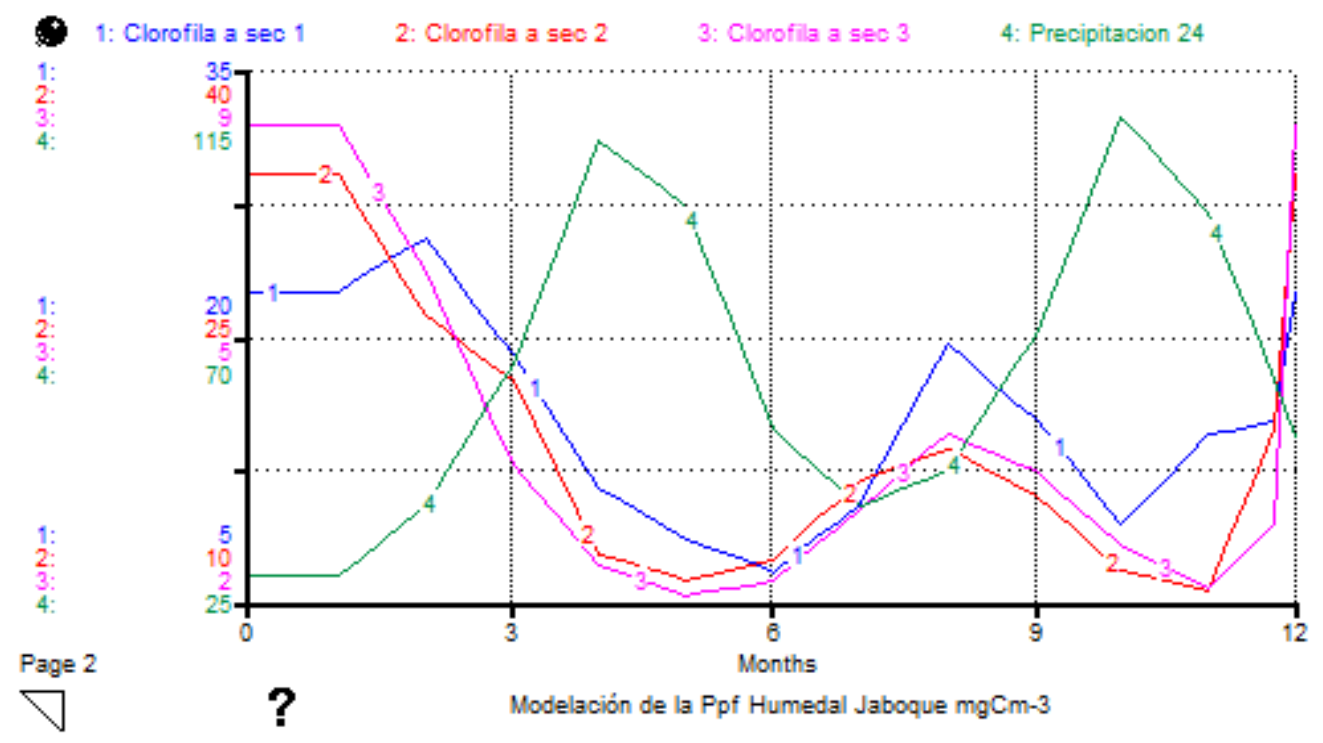

Figura 2. Resultado de la modelación de clorofila_a para el humedal de Jaboque, en las tres secciones

Fuente: elaboración propia.

1: Clorofila_a Sec $1 \mathrm{mg} / \mathrm{m}^{3}$.

2: Clorofila_a Sec $2 \mathrm{mg} / \mathrm{m}^{3}$.

3: Clorofila_a Ssec $3 \mathrm{mg} / \mathrm{m}^{3}$.

4: Precipitación mm año.

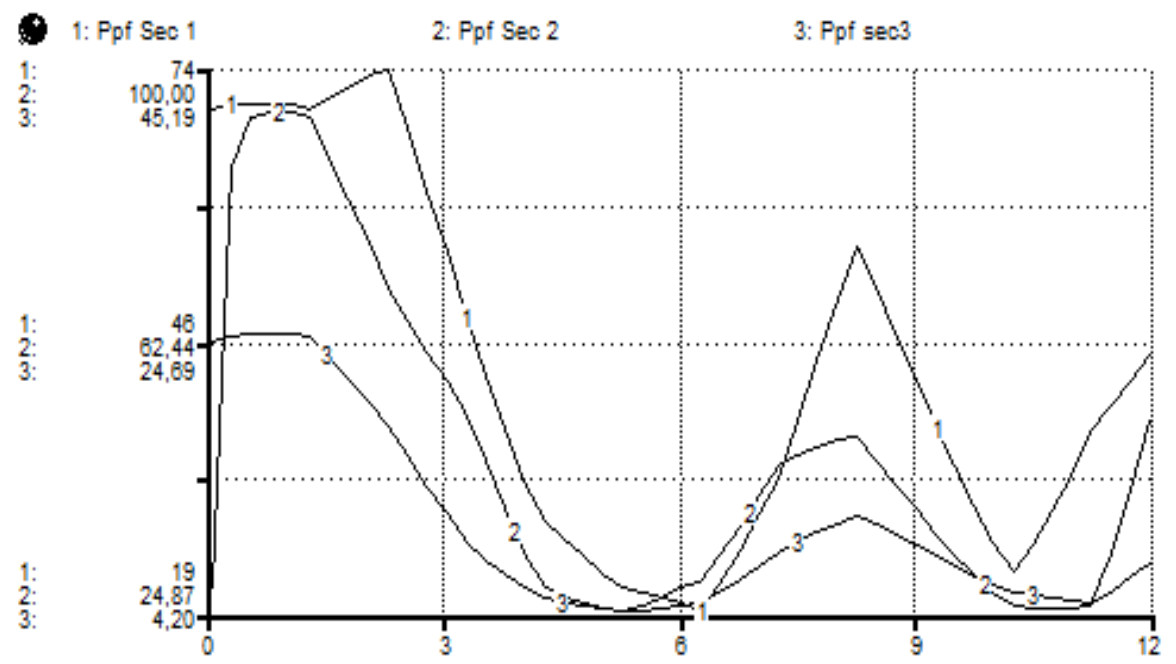

Figura 3. Modelación de la productividad primaria fitoplantónica para todas la secciones

Fuente: elaboración propia.

1: Producción primaria fitoplantónica Sec $1 \mathrm{mgCm}-3$ *año.

2: Producción primaria fitoplantónica Sec $1 \mathrm{mgCm}-3 *$ año.

3: Producción primaria fitoplantónica Sec $1 \mathrm{mgCm}-3$ *año. 
Sección 1. Zona intervenida (tercio alto)

En esta sección (figura 3 y tabla 3), durante los meses secos (enero, febrero, marzo) la producción primaria fluctuó entre $70,29 \mathrm{mgC} / \mathrm{m}^{-3}$ y $44,5 \mathrm{mgC} / \mathrm{m}^{-3}$, en los de agosto y septiembre entre $21,9 \mathrm{mCg} / \mathrm{m}^{-3}$, $39,21 \mathrm{mgC} \mathrm{m}^{-3}$; en diciembre fue de $36,8 \mathrm{mgC} / \mathrm{m}^{-3}$ y en los meses de mayores precipitaciones (abril, mayo y junio) los valores fluctuaron entre $28,5 \mathrm{mg}$ $\mathrm{C} / \mathrm{m}^{-3}$ y $16,7 \mathrm{mgC} / \mathrm{m}^{-3}$, y en octubre y noviembre, $23,3 \mathrm{mgC} / \mathrm{m}^{-3}$ y $29,53 \mathrm{mgC} / \mathrm{m}^{-3}$. El promedio mensual para toda la sección fue de $35,10 \mathrm{mgC} / \mathrm{m}^{-3} \mathrm{y}$ la desviación estándar fue de 15,77, lo cual indica que existe variación. La relación entre los valores de clorofila_a estimados con aquellos que se modelaron de la producción primaria fitoplantónica (Hakanson y Boulion, 2003; Kuo, Hsieh, Lung y She, 2007) se realizó un análisis de regresión y su resultados mostraron una buena correspondencia entre los dos grupos $r^{2}=0.85 ; y=0,3458 x+2,6623$.

\section{Sección 2. Zona de transición (tercio medio)}

En esta sección (figura 3 y tabla 3), en los meses secos (enero, febrero, marzo) la producción primaria fluctuó entre $77,8 \mathrm{mgC} / \mathrm{m}^{-3}$ y $50,02 \mathrm{mgC} / \mathrm{m}^{-3}$; durante agosto y septiembre estuvo entre $41,6 \mathrm{mC}$ $\mathrm{g} / \mathrm{m}^{-3}$ y $35,4 \mathrm{mgC} / \mathrm{m}^{-3}$; en diciembre fue de 31,5 $\mathrm{mgC} / \mathrm{m}^{-3}$; en los meses de mayores precipitaciones (abril, mayo y junio) los valores fluctuaron entre $32,2 \mathrm{mgC} / \mathrm{m}^{-3}$ y $24,8 \mathrm{mgC} / \mathrm{m}^{-3}$, mientras que en octubre y noviembre fue de $27,1 \mathrm{mgC} / \mathrm{m}^{-3}$ y 25 $\mathrm{mgC} / \mathrm{m}^{-3}$. El promedio mensual para toda la sección fue de 39,17 $\mathrm{mgC} / \mathrm{m}^{-3}$ y la desviación estándar fue de 16,73, mostrando que existe variación. La relación entre los valores estimados de clorofila con aquellos modelados de la producción primaria fitoplantónica mostraron que entre los dos grupos hay una buena correspondencia $r^{2}=0,8631$; $y=0,4043 x+0,8631$.

\section{Sección 3. Zona conservada (tercio bajo)}

En esta sección (figura 3 y tabla 3), en los meses secos (enero, febrero, marzo), la producción primaria fluctuó entre $15,9 \mathrm{mgC} / \mathrm{m}^{-3}$ y $12,5 \mathrm{mgC}^{-3} \mathrm{~m}^{-3}$; en agosto y septiembre estuvo entre $8,87 \mathrm{mCg} / \mathrm{m}^{-3} \mathrm{y}$ $7,98 \mathrm{mgC} / \mathrm{m}^{-3}$; en diciembre fue de $7,5 \mathrm{mgC} / \mathrm{m}^{-3}$; en los meses de mayores precipitaciones (abril, mayo y junio) los valores fluctuaron entre $5,61 \mathrm{mgC} / \mathrm{m}^{-3}$ y $4,16 \mathrm{mgC} / \mathrm{m}^{-3}$, y en octubre y noviembre fue de

Tabla 3. Resultados de la modelación de Chl_a y la Ppf

\begin{tabular}{|c|c|c|c|c|c|c|c|}
\hline Meses & Precipitación & $\begin{array}{c}\mathrm{Chl} \_\mathrm{a} \text { Section } 1 \\
\mathrm{mg} / \mathrm{m}^{3}\end{array}$ & $\underset{\mathrm{mgCm}^{-3}}{\text { Ppf Sección } 1}$ & $\underset{\substack{\mathrm{mg} / \mathrm{m}^{3} \\
\text { Chl_a Sección } 2}}{2}$ & $\underset{\mathrm{mgCm}^{-3}}{\text { Ppf Sección } 2}$ & $\begin{array}{c}\text { Chl_a Sección } 3 \\
\mathrm{mg} / \mathrm{m}^{3}\end{array}$ & $\begin{array}{l}\text { Ppf Sección } \\
\mathrm{mgCm}^{-3}\end{array}$ \\
\hline Enero & 29 & 22,5 & 70,29 & 34,2 & 77,8 & 7,8 & 12,5 \\
\hline Febrero & 41 & 25,5 & 55,67 & 26,1 & 63,51 & 5,85 & 15,96 \\
\hline Marzo & 65 & 18,9 & 44,5 & 22,5 & 50,02 & 3,3 & 9,72 \\
\hline Abril & 103 & 11,4 & 28,55 & 12,6 & 32,29 & 1,95 & 5,61 \\
\hline Mayo & 92 & 8,4 & 20,28 & 11,1 & 24,85 & 1,56 & 4,15 \\
\hline Junio & 54 & 6,6 & 16,02 & 12,3 & 25,73 & 1,74 & 4,16 \\
\hline Julio & 41 & 10,5 & 21,99 & 16,8 & 35,15 & 2,7 & 6,36 \\
\hline Agosto & 47 & 19,5 & 39,21 & 18,6 & 41,67 & 3,69 & 8,87 \\
\hline Septiembre & 70 & 15,3 & 34,98 & 15,9 & 35,39 & 3,21 & 7,98 \\
\hline Octubre & 107 & 9,3 & 23,35 & 11,7 & 27,12 & 2,22 & 5,92 \\
\hline Noviembre & 91 & 14,4 & 29,53 & 10,5 & 25 & 1,65 & 4,76 \\
\hline Diciembre & 53 & 15,3 & 36,85 & 22,8 & 31,5 & 2,8 & 7,5 \\
\hline Promedio & 66,08 & 14,80 & 35,10 & 17,93 & 39,17 & 3,21 & 7,79 \\
\hline $\begin{array}{l}\text { Desviación } \\
\text { estándar }\end{array}$ & 26,39 & 5,89 & 15,77 & 7,28 & 16,73 & 1,87 & 3,57 \\
\hline
\end{tabular}

Fuente: elaboración propia. 
$5,92 \mathrm{mgC} / \mathrm{m}^{-3}$ y $4,76 \mathrm{mgC} / \mathrm{m}^{-3}$. El promedio mensual para toda la sección fue de $7,79 \mathrm{mgC} / \mathrm{m}^{-3}$ y la desviación estándar fue de 15,77, mostrando que existe variación. La relación entre los valores de clorofila_a estimados con aquellos modelados de la producción primaria fitoplantónica (Hakanson y Boulion, 2003; Kuo, Hsieh, Lung y She, 2007) se realizó mediante análisis de regresión, y sus resultados mostraron que entre los dos grupos hay una buena correspondencia $r^{2}=0,792 ; y=1,6947 x+2,358$.

Los resultados anteriores indican que la clorofila_a y la producción primaria fitoplantónica se relacionan con el hidroperiodo y el transporte de nutrientes como lo sugieren Ryding y Rast (1989). Pinilla (2006) encontró un patrón de comportamiento similar en la laguna de Guatavita (Cundinamarca, Colombia) y un lago amazónico (Hernandez, Aguirre, Palacio y Ramírez, 2008) hallaron que en la ciénaga de Ayapel (Córdoba, Colombia), los nutrientes y la clorofila_a se encuentran regulados por el hidroperiodo y el pulso de la inundación.

\section{Error relativo}

Para verificar la capacidad de predicción o fortaleza del modelo se determinó el error relativo (Bryhn y Hakanson, 2007; Jorgensen y Bendoricchio, 2001), para cada una de las secciones, para lo cual se compararon los valores medidos, clorofila_a con los valores resultante de la modelación de la Ppf. El valor promedio del error relativo para el tercio alto es de 1,36, para el segundo tercio de 1,21 , y para el tercero, de 1,56; y las desviaciones estándar fueron de 0,30 para el primer tercio, 0,29 para el segundo y el tercero de 0,36 , cifras que indican que el modelo no tiende a sobreestimar los valores es su predicción.

\section{Estado trófico (IST) basado en la producción primaria}

Los resultados de la modelación permitieron establecer las cantidades de clorofila_a, debido a que esta es proporcional a la cantidad de fitoplancton y esta es a su vez proporcional al estado trófico del cuerpo de agua de acuerdo con Hakanson y Boulion, (2003) y Ryding y Rast (1989), se utilizaron para determinar el estado trófico de cada una de las secciones del humedal (tablas 1 y 3 ). Los resultados anteriores permiten inferir que a mayores descargas de agua de la cuenca aferente es posible disminuir las condiciones de eutrofia en las dos primeras secciones, y en la tercera sección mejorar o disminuir la tendencia a la mesotrofia, como lo sugiere Jorgensen (2003) que al aumentar la descarga de agua se presenta un lavado de nutrientes, mejorando las condiciones tróficas, fenómeno similar al observado con el comportamiento del fósforo total en el humedal.

\section{Análisis de sensibilidad del modelo}

Para el análisis de sensibilidad del modelo se tuvo en cuenta el promedio anual de la clorofila_a en $\mathrm{mg} / \mathrm{m}^{3}$ como variable independiente expresada en clases entre $4,0 \mathrm{mg} / \mathrm{m}^{3}$ y $18 \mathrm{mg} / \mathrm{m}^{3}$ y como variable dependiente la Ppf expresada en $\mathrm{mgCm}^{-3}$. En la figura 4 se aprecian los resultados de análisis de sensibilidad, para la tercera sección. Las curvas de la figura corresponden a los cambios en la Ppf según cada nivel de variación de los valores de clorofila_a entre clases desde $4 \mathrm{mg} / \mathrm{m}^{3}$ a $18 \mathrm{mg} / \mathrm{m}^{3}$; las coordenadas señalan el tiempo en meses, y la abscisa a los valores de Ppf en $\mathrm{mgCm}^{-3}$. Los resultados permitieron corroborar la correspondencia entre los valores de clorofila_a y la Ppf, a medida que aumentan las cantidades de clorofila_a aumenta la Ppf, como lo sugieren Wetzel (2001); Gocke, Mancera, Vidal y Fonseca, 2003). Se percibe una fuerte influencia del ciclo hidrológico en el comportamiento de la marcha de las dos variables, en las épocas más lluviosas (abril, mayo, junio y octubre, noviembre) la Ppf disminuye y aumenta en los periodos secos, como ocurre en el humedal Jaboque (Álvarez, 2005). Esto se debe, en parte, a la oferta de nutrientes que se concentra en las épocas de verano cuando disminuye el volumen de agua y la concentración se diluye cuando aumenta el 


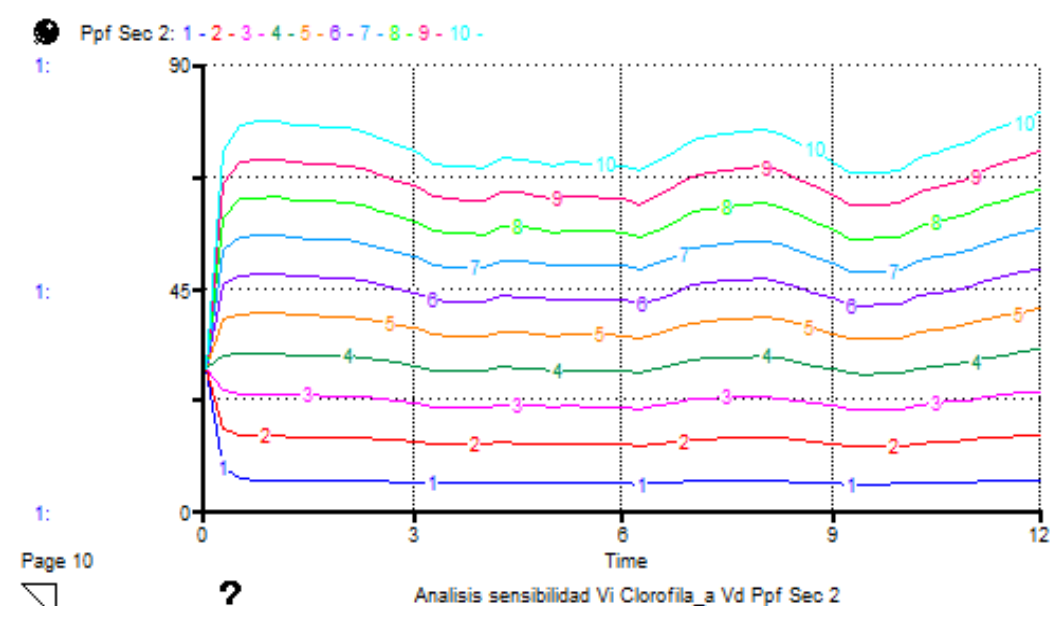

Figura 4. Análisis de sensibilidad. Tercio bajo. Variable independiente clorofila_a, variable dependiente Ppf

Fuente: elaboración propia.

Ppf sec1: productividad primaria fitoplántonica $\mathrm{mgCm}-3$ año.

$1,2,3 \ldots$. niveles de variación clorofila_a entre $4 \mathrm{mg} / \mathrm{m} 3$ y $18 \mathrm{mg} / \mathrm{m} 3$.

volumen de agua. Los resultados anteriores siguieren que para mantener una condición mesotrófica en el humedal, el máximo permisible correspondería a $11,8 \mathrm{mg} / \mathrm{m}^{3}$ de clorofila_a con una Ppf de $29,1 \mathrm{mgCm}^{-3}$.

\section{CONCLUSIONES}

Las dos primeras secciones muestran las mayores concentraciones de clorofila_a, mientras que la tercera sección las concentraciones de clorofila_a tienden a disminuir, resultado que se debe al hecho de que la primera y segunda sección reciben con mayor fuerza la descarga de nutrientes de la cuenca aferente; sin embargo la segunda sección presenta los mayores valores de clorofila_a. En la tercera sección las concentraciones disminuyen de manera significativa, indicado mejor condición trófica, como lo señala Álvarez (2005). Los resultados de la modelación permitieron corroborar que las cantidades de clorofila_a son proporcionales a la Ppf y esta a su vez es proporcional al estado trófico del humedal. Se encontró dependencia entre el ciclo hidrológico y el comportamiento de la producción primaria fitoplantónica; como lo indica
Álvarez (2005), este fenómeno es más acentuado en el tercio alto y disminuye gradualmente hacia al tercio bajo. Estos resultados corroboran la relación entre la clorofila_a y la producción primaria fitoplantónica como lo sugieren Ryding y Rast (1989) y como los reportados para la ciénaga de Ayapel (Córdoba, Colombia) por Hernández, Aguirre, Palacio y Ramírez (2008).

La bondad de predicción del modelo se verificó mediante la determinación del error relativo con los siguientes valores: 1,36 y 0,3 de desviación estándar en el primer tercio; 1,21 para el segundo tercio, y 0,29 de desviación estándar; y en la tercera 1,56 y 0,36 de desviación, valores cercanos a cero. La correspondencia entre las variables del modelo entre los valores de clorofila_a y aquellos simulados de Ppf calculados, mostraron una alta reciprocidad, para el tercio alto de $r^{2}=$ 0,86 ; el tercio medio $r^{2}=0,86$; el tercio bajo $r^{2}=$ 0,78 , respectivamente. Si bien hay diferencias, es posible que se deban al complejo patrón de distribución de nutrientes y así mismo de los procesos biogeoquímicos en el humedal (Álvarez, 2005). El análisis de sensibilidad del modelo permitió comprobar la relación entre una variable física como 
es la precipitación y la correspondencia entre una variable química la clorofila_a y una de tipo biótico la producción primaria, mostrando el carácter holístico e integrador del modelo (Carpenter y Cottingham, 1997; Boumans et al., 2002). También se evidenció la correspondencia entre las variables de entrada y salida del modelo, y su carácter predictivo. Además se demostró que la estructura y las variables del modelo reproducen de manera general el comportamiento de la producción primaria fitoplantónica del humedal Jaboque.

De acuerdo con los resultados de la modelación se puede concluir que en el humedal Jaboque la producción primaria es alta en las dos primeras secciones y especial en los meses de menores precipitaciones; en la tercera sección, la productividad primaria disminuye de manera significativa marcando una diferencia clara entre un estado mesotrófico y el estado eutrófico de las dos primeras secciones. La modelación permitió comprobar la bondad de utilizar indicadores de estado trófico, mostrando el potencial que tienen este tipo de indicadores en la vigilancia y control de la eutroficación.

\section{FINANCIAMIENTO}

Universidad Distrital Francisco José de Caldas Rectoría, Facultad del Medio Ambiente y Recursos Naturales, Centro de Investigaciones y Desarrollo Científico (CIIDC).

\section{AGRADECIMIENTOS}

Al Grupo de Investigación Biodiversidad y Conservación de la Universidad Nacional de Colombia Instituto de Ciencias Naturales, y a la Empresa de Acueducto y Alcantarillado de Bogotá (EAAB-ESP), por el aporte de la información relacionada con el estudio de caso. A la Universidad Distrital Francisco José de Caldas - Rectoría, la Facultad del Medio Ambiente y Recursos Naturales por el apoyo económico otorgado, al Centro de Investigaciones y Desarrollo Científico (CIIDC) y el Grupo de Investigación INDESOS, quienes apoyaron la financiación para la adquisición de los programas computacionales, el equipo de cómputo y la bibliografía especializada que se utilizó en la investigación.

\section{REFERENCIAS}

Álvarez, J. (2005). Evaluación del estado trófico del humedal de Jaboque: análisis espacial y temporal de las características fisicoquímicas del agua y de la comunidad planctónica. En: Informe final. Investigación aplicada en restauración ecológia en el humedal de Jaboque (pp. 215-235). Bogotá: Convenio Acueducto de Bogotá-Universidad Nacional de Colombia.

Boulion , V. \& Hakanson, A. (2003). A New General Dynamic Model to Predict Biomass and Production of Bacterioplankton in Lakes. Ecological Modelling 160(2), 91-114.

Boumans, R.; Constanza, R.; Farley, J.; Wilson, M.; Portela, R.; Rotmans, J.; Villa, F. y Grasso, M. (2002). Modeling the Dynamics of the Integrated Earth System and the Value of Global Ecosystem Services Using the GUMBO Model. Ecological Economics 41(3), 529-560.

Bryhn, A. \& Hakanson, L. (2007). A Comparison of Predictive Phorphorus Load-Concentration Models for Lakes. Ecosystems 10(7),1084-1099.

Carpenter, S. \& Cottingham, K. (1997). Resilience and Restoration of Lakes. Conservation ecology 1(1), 2-3

Contreras, F.; Castañeda, O. y Torres, R. (1997). Hidrología, nutrientes y productividad primaria en las lagunas costeras del estado de Oaxaca, México. Hidrobiológica 7(1), 9-17.

Fraile, H.; Orive, E. y Pozo, J. (1995). Evaluación del estado trófico y comparación de modelos relativos al fósforo en los embalses de Cernadilla y Valparaiso (río Tera y Zamora). Limnetica 11(2), 29-37.

Gocke, K.; Mancera, P.; Vidal, L. \& Fonseca, D. (2003). Planktonic Primary Production and Community Respiration in Several Coastal Lagoons of the Outer Delta of the Rio Magdalena, Colombia. Boletín de investigaciones marinas y costeras 32(1), 125-144.

Gómez, L.; Larduet, Y. y Abrahantes, N. (2001). Contaminación y biodiversidad en ecosistemas acuáticos. 
El fitoplancton en la bahía de Santiago de Cuba. Rev. Invest. Mar. 22(3), 191-197.

Grimshaw, H.; Wetzel, R.; Brandenburg, M.; Segerblom, K.; Wenkert, L.; March, G.; Charnetzky,W.; Haky, J. \& Carraher, C. (1997). Shading of Peryphyton Communities by Wetland Emergent Macrophytes: Decoupling of Algal Photosynthesis from Microbial Nutrient Retention. Archiv für Hydrobiologie 139(1), 17-27.

Hakanson, L. (2004). Break-Throught in Predictive Modelling Opens new Possibilities for Aquatic Ecology and Management-A Review. Hydrobiologia. Volume 518(1-3), 135-157.

Hakanson, L. \& Peters, R.H. (1995). Predictive Limnology: Methods for predictive modelling. Amsterdam: SPB Academic Publishing.

Hakanson, L. \& Boulion, V. (2002). Empirical and Dynamical Models to Predict the Cover, Biomass and Production of Macrophytes in Lakes. Ecological Modelling 151(2), 213-243.

Hakanson, L. \& Boulion, V. (2003). A General Dynamic Model to Predict Biomass and Production of Phytoplankton in Lakes. Ecological Modelling 165(2), 285-301.

Hernandez, E.; Aguirre, J.; Palacio, J. y Ramírez, J. (2008). Variación espacio temporal de la asociacion fitoplantónica en diferentes momentos del pulso hidrologico en la ciénaga de Ayapel, Cordoba. Colombia actualidades biológicas 30(88),67-81.

Jiménez, H. (2006). Las variaciones diarias de la producción primaria y la concentración de la clorofila en un lago tropical (Guatavita, Colombia). Tesis inédita de maestría. Bogotá, Colombia: Pontificia Universidad Javeriana.

Jorgensen, S.E. (2003). The Aplication of Models to Find the Relevance of Residence Time in Lake and Reservoir Management. Journal of limnogy 62 (1),16-20.

Jorgensen, S. (2008). Overview of the Model Types Available for Development of Ecological Models. Ecological Modelling 215(1), 3-9.
Jorgensen, S. \& Bendoricchio, G. (2001). Fundamentals of ecological modelling. Ámsterdam: Elsevier Science Ltda.

Kuo, J.T.; Hsieh, M.H.; Lung, W.S. y She, N. (2007). Using Artificial Neural Network for Reservoir Eutrophication Prediction. Ecological Modelling 200(1), 171-177.

Legendre, P. y Legendre, L. (1998). Numerical ecology. Ámsterdam: Elsevier Science.

Montoya, Y. y Aguirre, N. (2010). Dinámica de la producción primaria fitoplantónica en un lago tropical (ciénaga Escobilletas) a lo largo del pulso de inundación (pp. 76-89). Medellín: Facultad de Ingenieria de la Universidad de Antioquia,

Pinilla, G. (2006). Evaluación de la eficiencia fotosintética del fitoplancton en un lago amazónico (lago Boa) y en un lago andino (lago Guatavita). Acta Amazónica 36(2), 221-228.

Rivera, C. (2006). Modelos correlativos de algunas variables físicas, químicas y biológicas del lago Guatavita. Tesis de maestría. Bogotá: Pontificia Universidad Javeriana.

Ryding, S.O. \& Rast, W. (1989). Control of Eutrophication of Lakes and Reservoirs. Manual the Biophere Series. Vol. 1. Nashville: Parthenon Publishing Group.

Sierra, O.R. \& Monsalve, C.A. (2005). Zonificación trófica del humedal Jaboque, con base en la familia Bacillariophyceae, Engativá. Investigacion aplicada en restauración ecológica en el humedal de Jaboque. Bogotá: Acueducto de Bogotá, Universidad Nacional de Colombia.

Wetzel, R. (2001). Fundamental Processes Within Natural and Constructed Wetland Ecosystems: ShortTerm Versus Long-Term Objectives. Water Science \& Technology 44(11-12), 1-8.

Zhang, L. \& Mitsch, W. (2005). Modelling Hydrological Processes in Created Freshwater Wetlands: An Integrated System Approach. Environmental Modelling \& Software 20(7), 935-946.

\section{(C) $(1) \Theta$}


